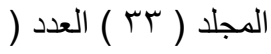

r...o(r

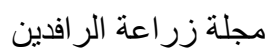

تأثير التراكيز المختلفة من تريكارد وطريقة المعاملة ودرجة حرارة التربية في النشاط الحيوي لخنفساء اللوبيا الجنوبية المرباة على بذور المعام الحمص

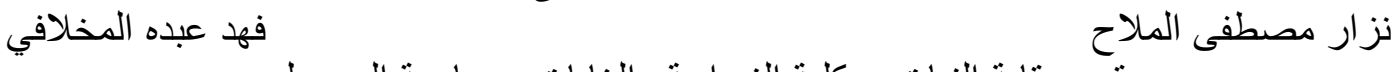

\title{
الخلاصة - - ماصة
}

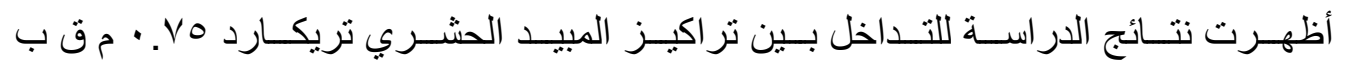

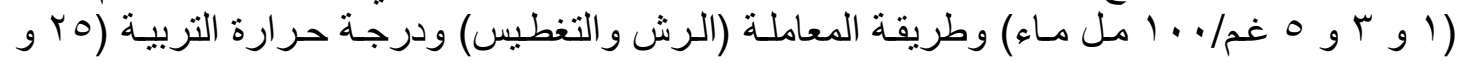

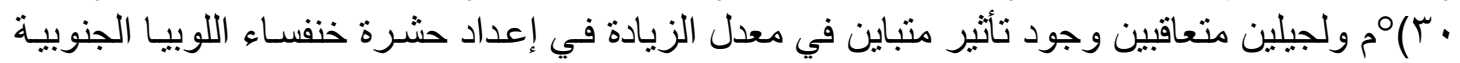
Callosobruchus maculatus (Fab.)

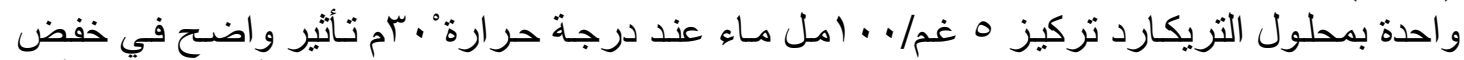

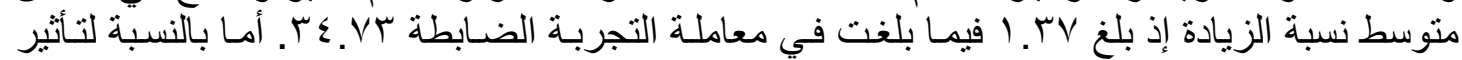

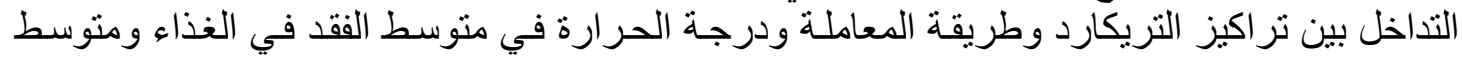

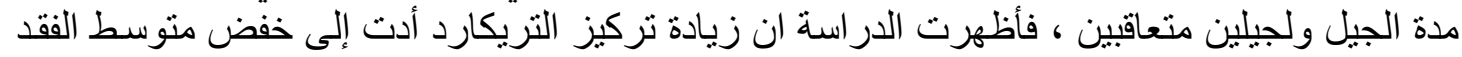

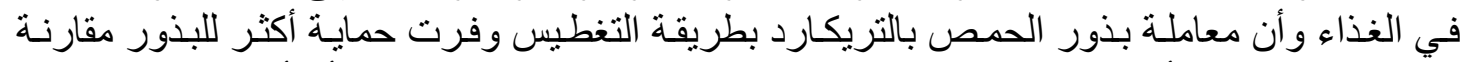

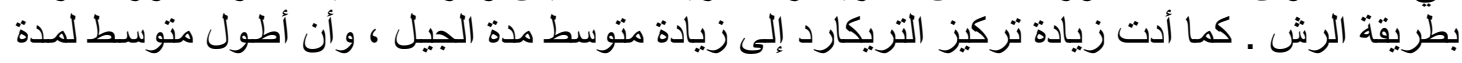

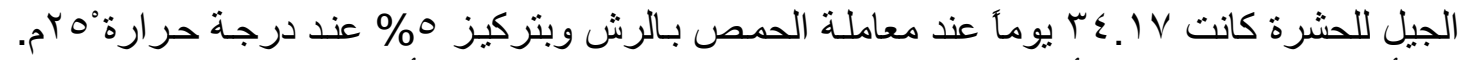

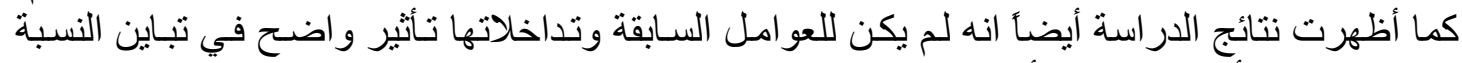

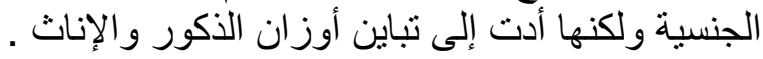

\section{المقدمة}

تعد خنفساء اللوبيا الجنوبية (Fallosobruchus maculatus (Fab. تلهوبة واحدة من أكثر الآفات

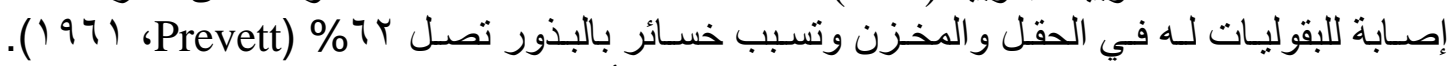

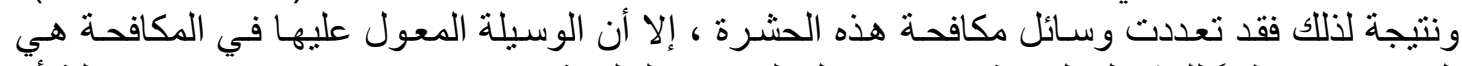

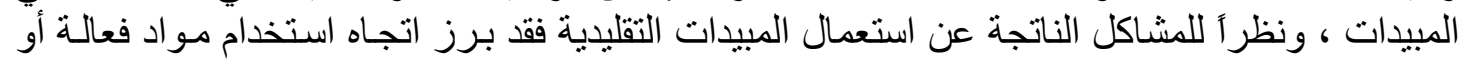

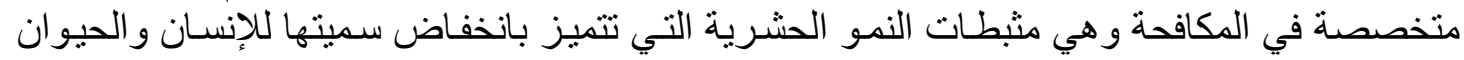

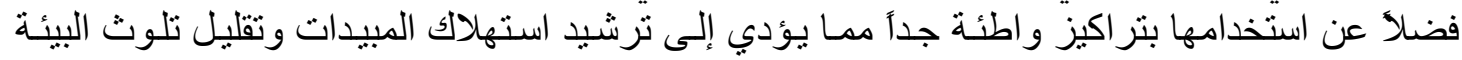

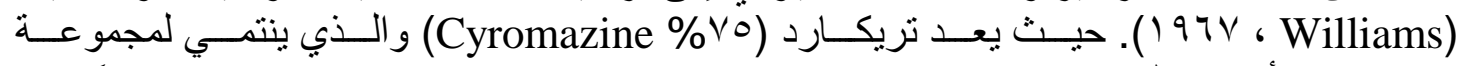
و وأســمه الكيميــائي T-Cyclopropyl-1,3,5-triazine-2,4,6-triamine مثبطات النمو الحشرية الجيدة والذي استخدمت بنجاح لمكافحة العديد من الآفات الحشـرية التابعـة لرتبـة التبة

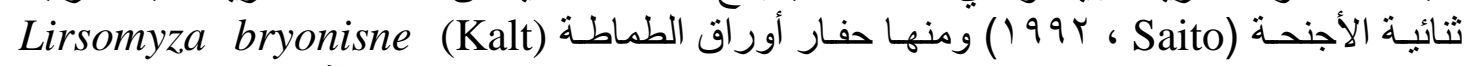

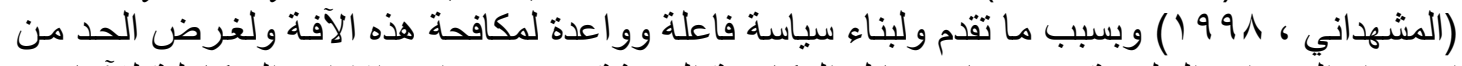

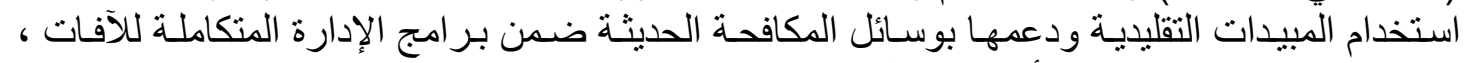

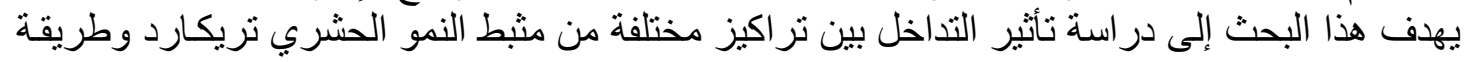

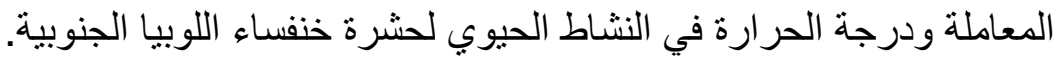

\section{مواد وطرق البحث}

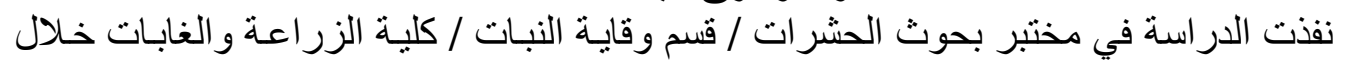

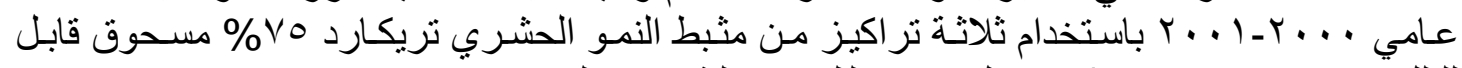

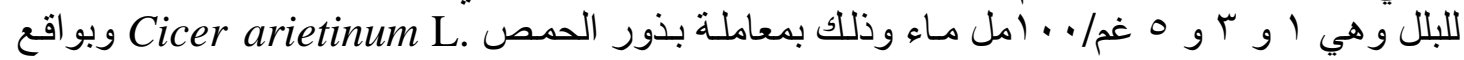

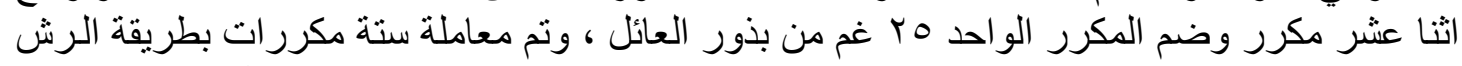

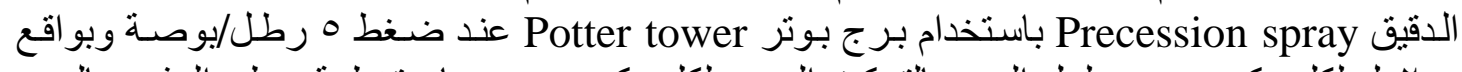
๑. rمل لكل مكرر من محلول المبيد بالتركيز المحدد لكل مكرر مع ضمان تغطية سطح البذور بالمبيد 
بشكل متجـانس فيمـا عوملت الستة الباقيـة من المكرر ات بغمرهـا في محلول المبيد لمدة دقيقة واحدة ( (19V1 ، Busvine)

$$
\begin{aligned}
& \text { البحث مستل من رسالة الماجستير للباحث فهر عبده المخلافي. }
\end{aligned}
$$

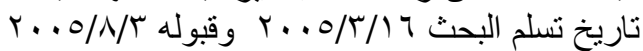

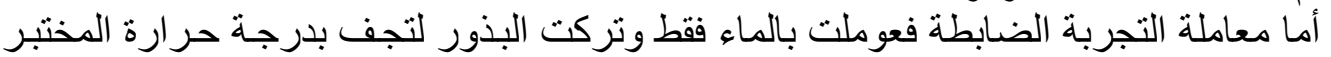

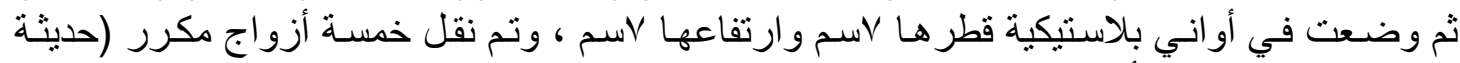

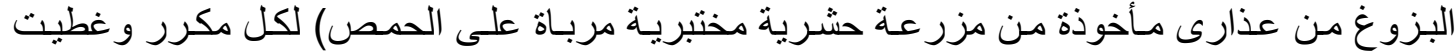

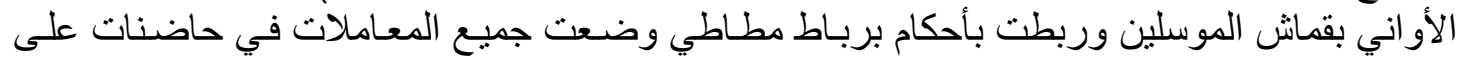

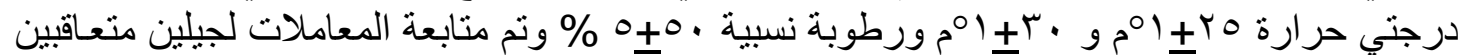

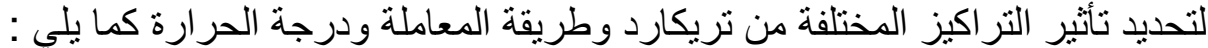

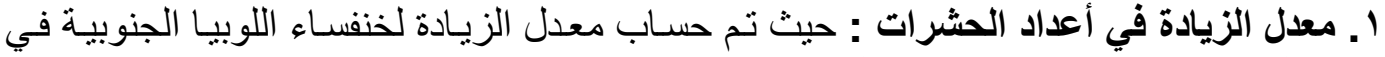

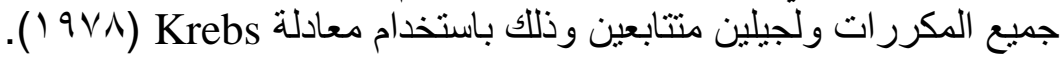
$\mathrm{dn} / \mathrm{dt}$

$\overline{\bar{n}}$

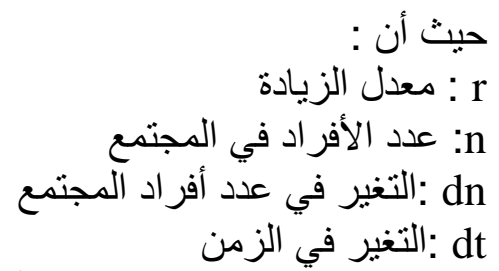

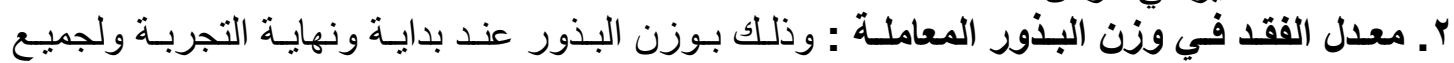

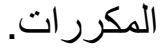

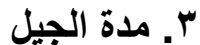
ع. النسبة الجنسية وأوزان الذكور والإناث : وذللك بأخذ مجمو عة عشوائية من الحشر ات الكاملـة لكل

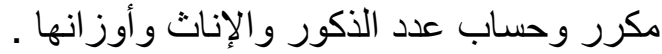
حلت النتائج إحصائياً باستخدام تصميم التجربة العاملية العشوائية الكاملـة التهائ (FCRD) واستخدم

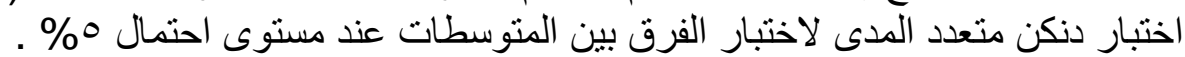

\section{النتائج و المناقشة}

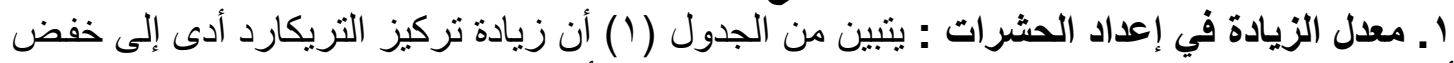

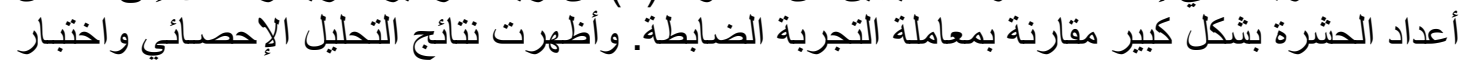

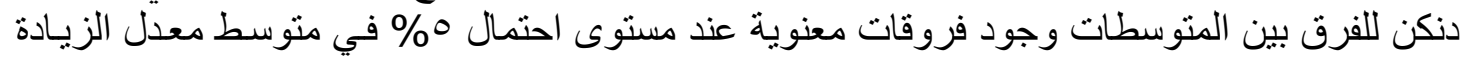

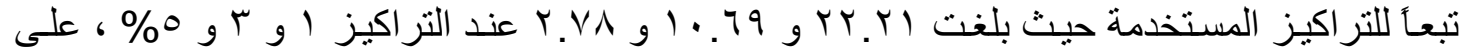

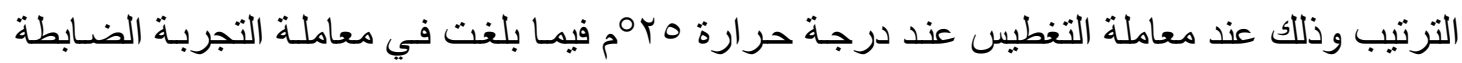

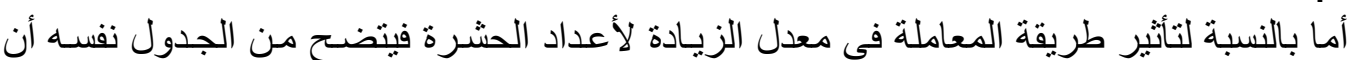

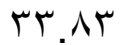

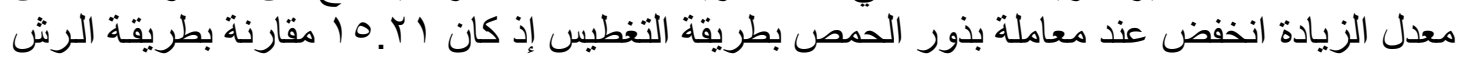

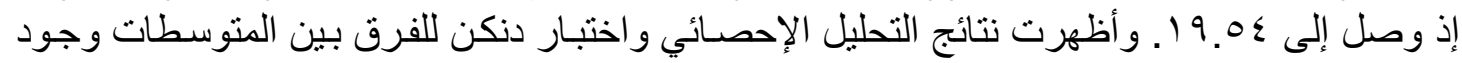

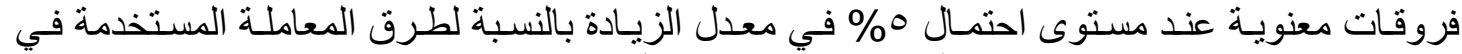

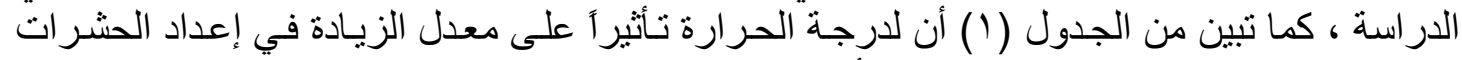

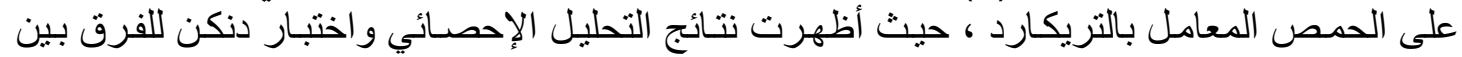

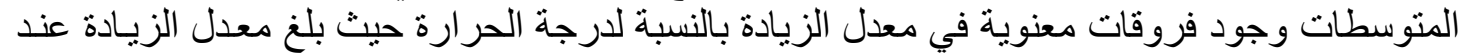

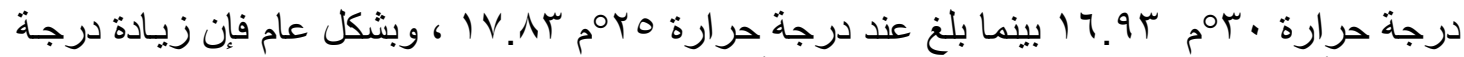

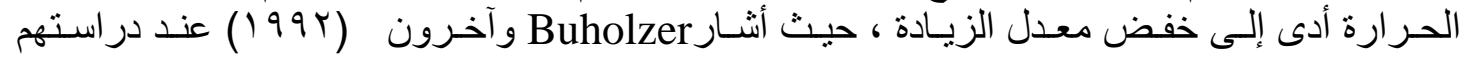

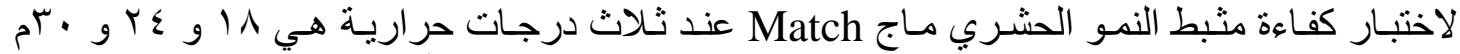

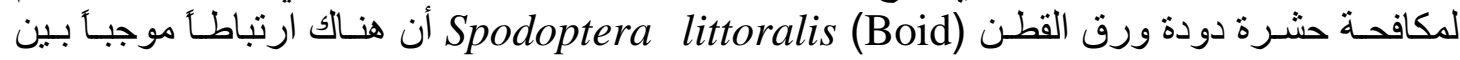




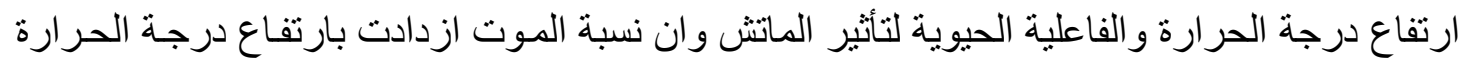

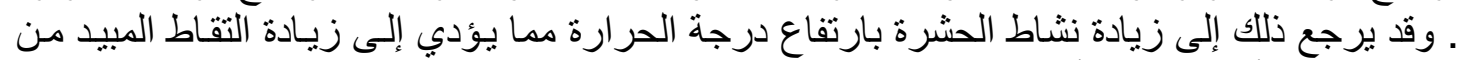

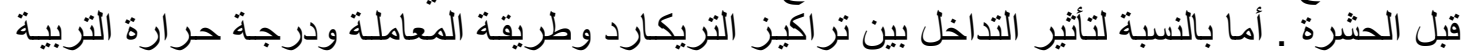

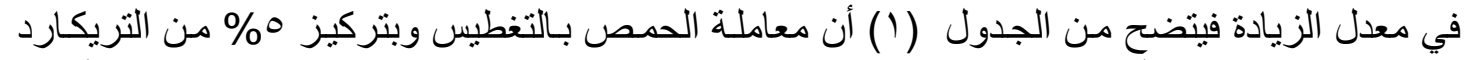

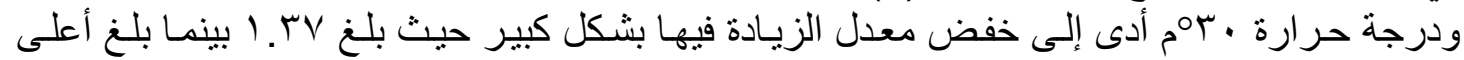

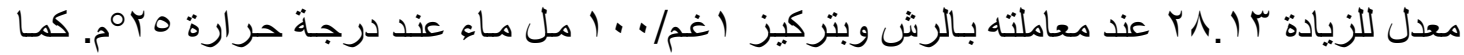

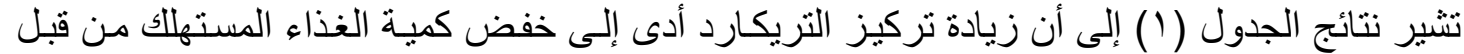

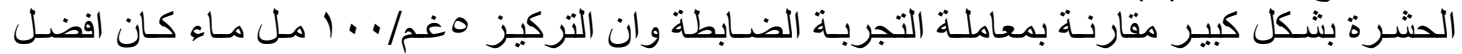

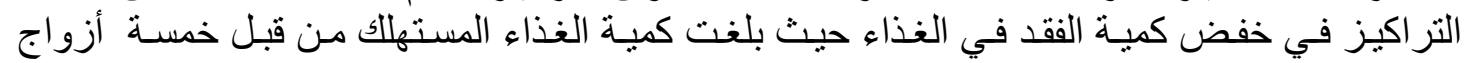

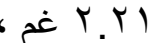

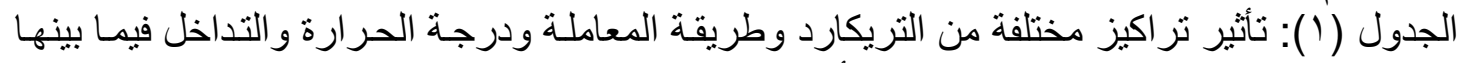
في منوسط معدل الزيادة في أعداد الحشرة ومنتوسط منس الفقد في الغذاء لجيلين متعاقبين

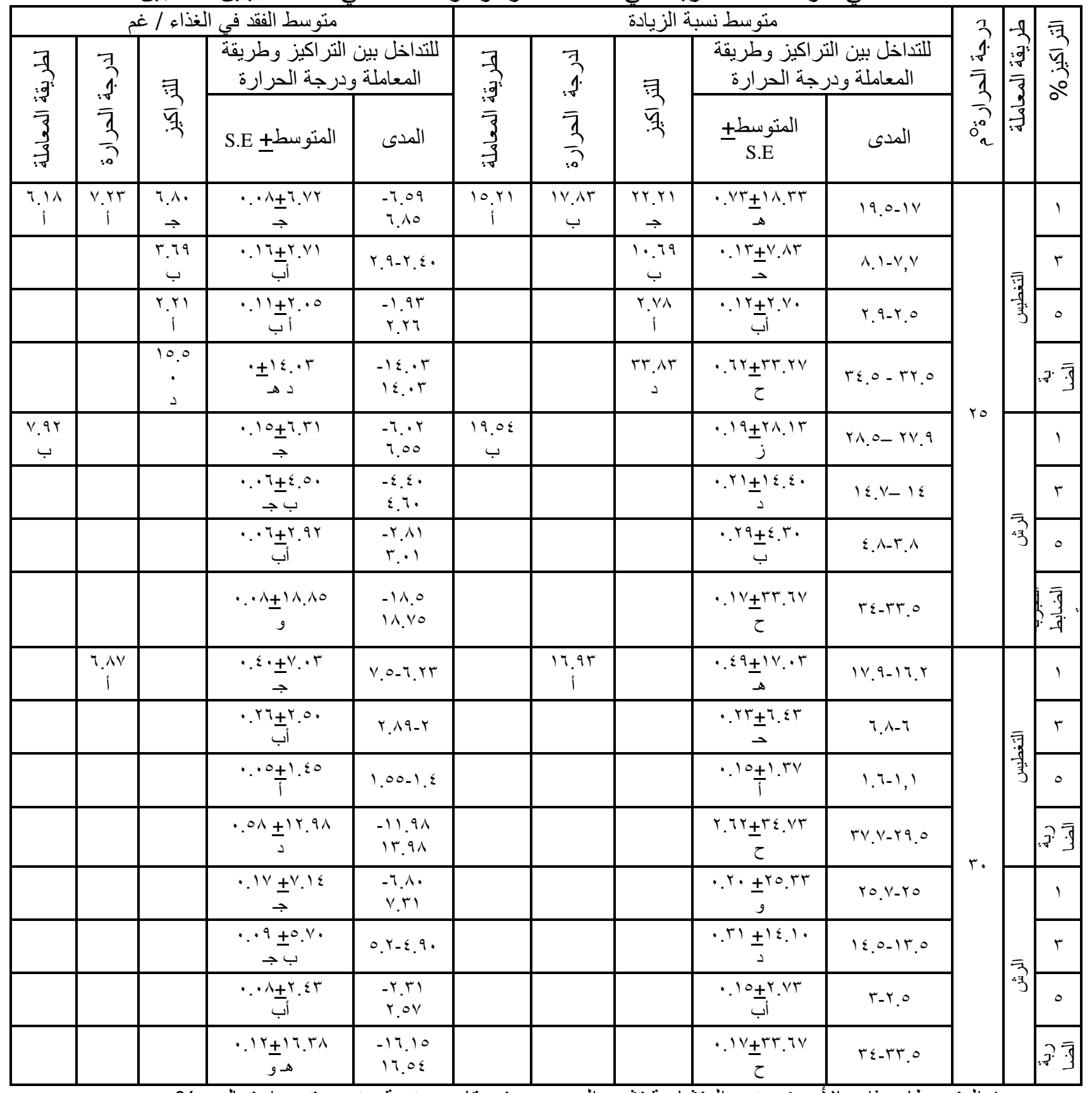

* المتوسطات ذات الأحرف غير المتشابهة تثير إلى وجود فروقات معنوية عند مستوى احتمال ه\%\%. 
في حين بلغت 0.0 أ غم في معاملة التجربة الضابطة وذلك عند المعاملة بالتغطيس و عند درجة حرارة

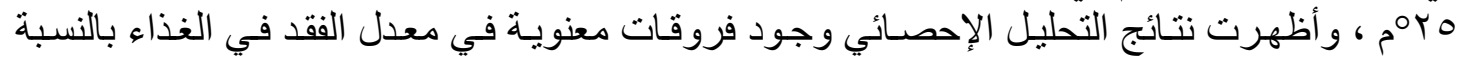

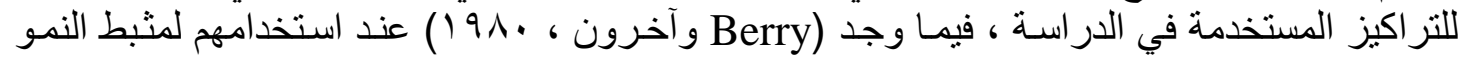

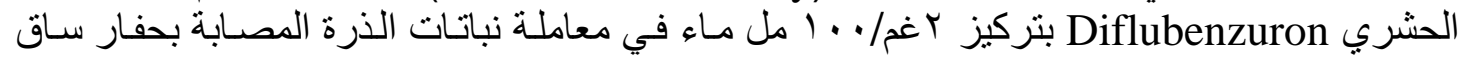

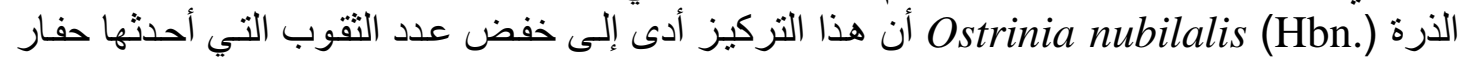
ساق الذرة مقارنة بالنباتات غير المعاملة.

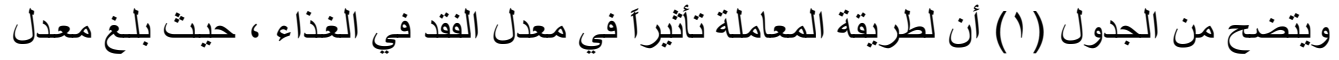

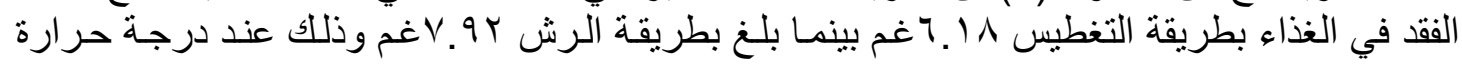

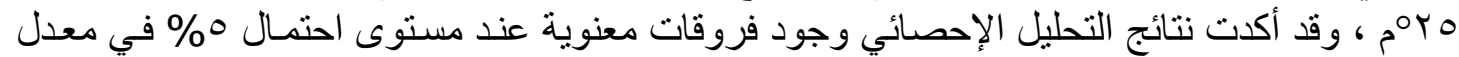

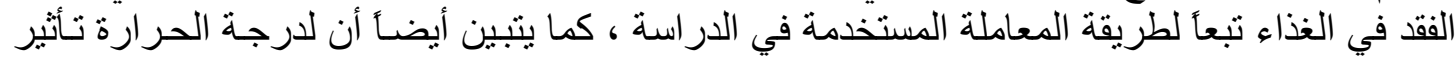

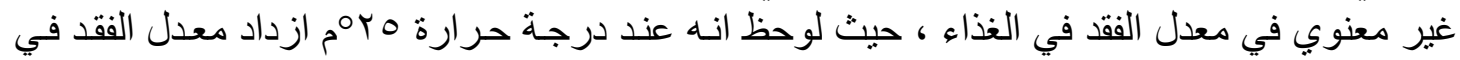

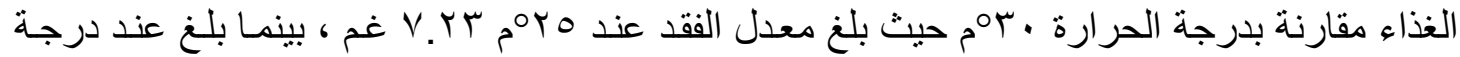

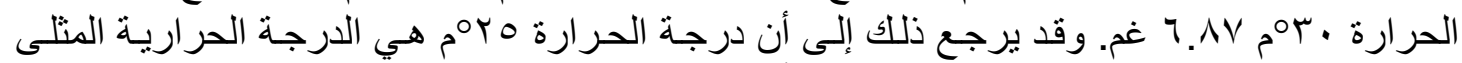

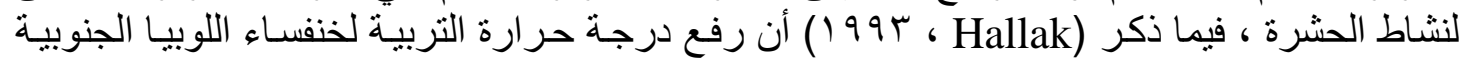

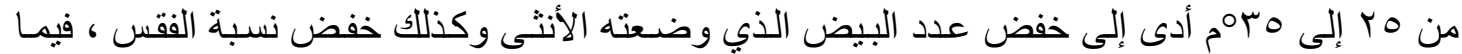

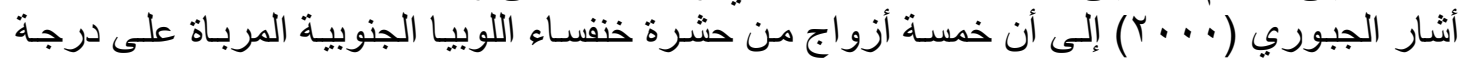

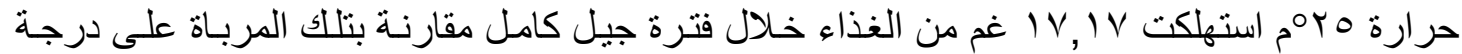

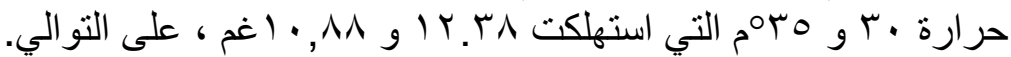

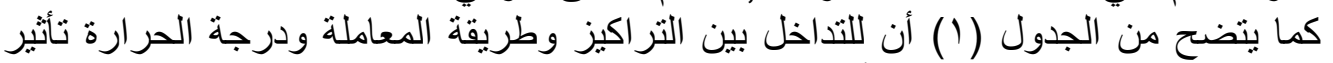

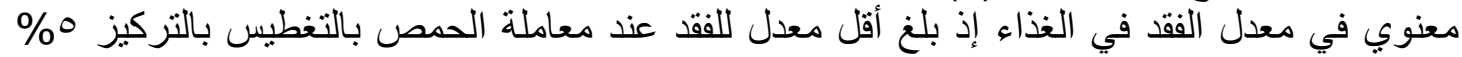
0.

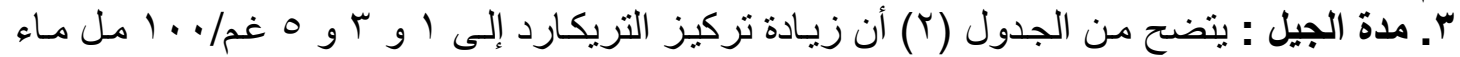

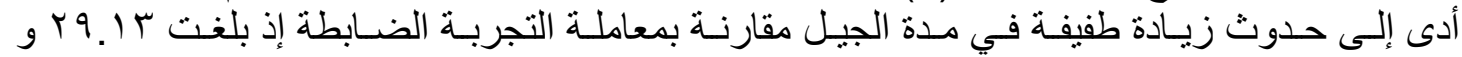

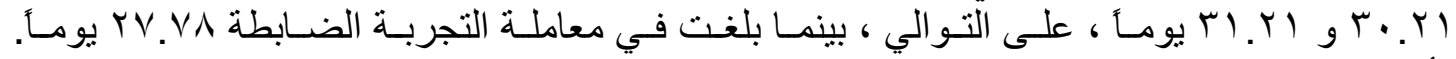

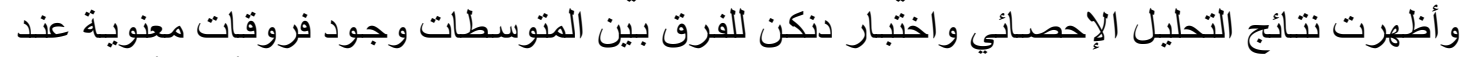

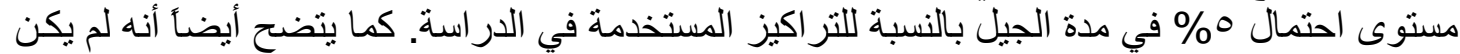

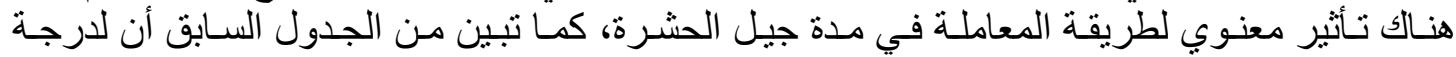

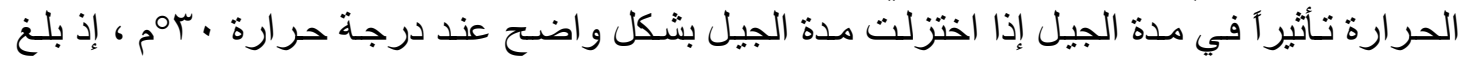

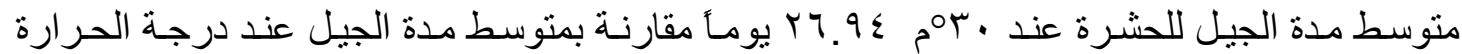

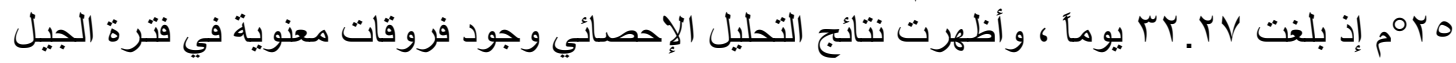

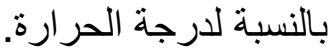
أما بالنسبة لتأثير التداخل بين تر اكيز التريكارد وطريقة المعاملة ودرجة الحرارة في متوسط مدة الجيل

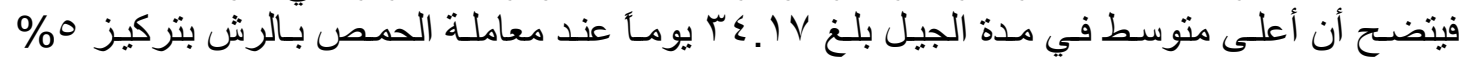

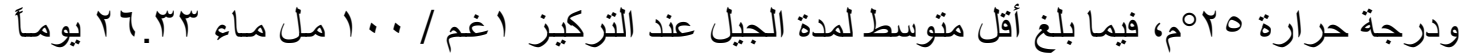

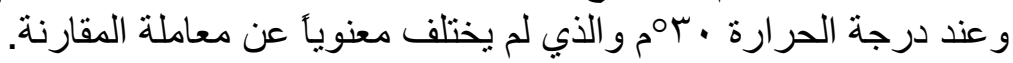

الجدول (Y): تأثثر تر اكيز مختلفة من تريكارد وطريقة المعاملـة ودرجـة الحر ارة والتداخل فيمـا بينها في المتوسط العام لمدة الجيل للحشرة لجيلئ تيلين متعاقبين

\begin{tabular}{|c|c|c|c|c|c|c|c|}
\hline \multicolumn{5}{|c|}{ متوسط فترة الجيل/يوم } & \multirow{3}{*}{ الحر ارجة } & \multirow{3}{*}{ المعاملة } & \multirow{3}{*}{ التر اكيز\% } \\
\hline \multirow{2}{*}{ 青亨 } & \multirow{2}{*}{ ᄀᄀ } & \multirow{2}{*}{ 寻. } & \multicolumn{2}{|c|}{ للتداخل بين التر اكيز وطريقة المعاملة } & & & \\
\hline & & & المنوسط+ S.E & الددى & & & \\
\hline$i r 9.0$. & $\overline{I r r . Y v}$ & جY9.1 & 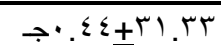 & TY-T. & ro & التغطيس & $T$ \\
\hline
\end{tabular}




\begin{tabular}{|c|c|c|c|c|c|c|c|}
\hline & & ון.r. & & Tr.0-TI.0 & & & $r$ \\
\hline & & |r. & $i \cdot r^{q} \pm r \leq$ & $T \leq .0-T T .0$ & & & 0 \\
\hline & & STV.AV & $د \cdot \pm r$. & $r \cdot-r$. & & & التجربة الضابطة \\
\hline \multirow[t]{12}{*}{ irq.V) } & & & & TY.O-TY.O & & \multirow{4}{*}{ 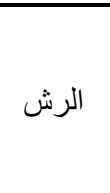 } & 1 \\
\hline & & & 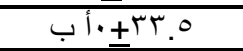 & 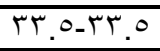 & & & $r$ \\
\hline & & & $1 \cdot . r T \pm r \leqslant .1 V$ & $r \leq .0-r T .0$ & & & 0 \\
\hline & & & $د \cdot .1 v_{ \pm} r \cdot .1 V$ & r. & & & التجربة الضابطة \\
\hline & (Y).9 & & $\tau j \cdot r r \pm r u . r r$ & $r V-r T$ & \multirow{8}{*}{ r. } & \multirow{4}{*}{ التغطيس } & 1 \\
\hline & & & $g-. r^{q}{ }^{q} \pm Y r_{.0}$ & YA-YV & & & $r$ \\
\hline & & & $\Delta \cdot .0 \pm Y \wedge .0$ & Y9.0-YA & & & 0 \\
\hline & & & $\tau \cdot . \Sigma \varepsilon \pm Y^{0} . \wedge r$ & YT.O-YO & & & التجربة الضـابطة \\
\hline & & & $\tau j \cdot r r \pm r q . r r$ & $r V-Y T$ & & \multirow{4}{*}{ 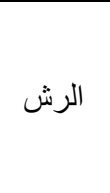 } & 1 \\
\hline & & & $j g . \varepsilon \varepsilon \pm Y V . r r$ & YV-YT.O & & & $r$ \\
\hline & & & 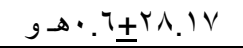 & Yq-YV & & & 0 \\
\hline & & & $\tau . .19 \pm r^{0.0}$ & YT-YO & & & التجربة الضابطة \\
\hline
\end{tabular}

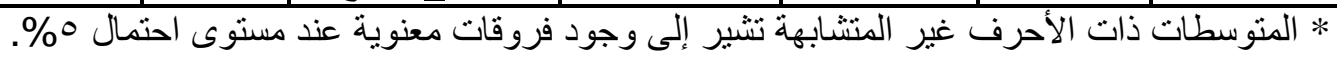

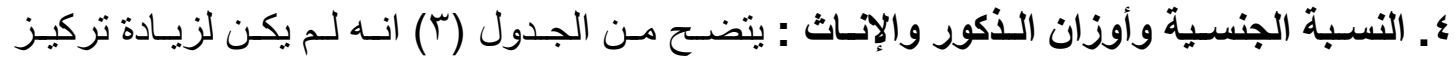

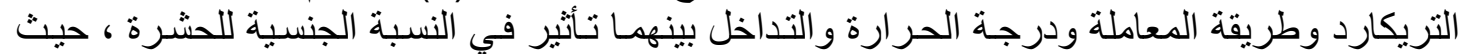

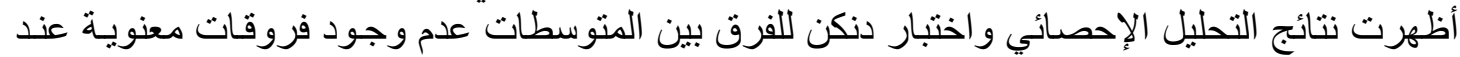

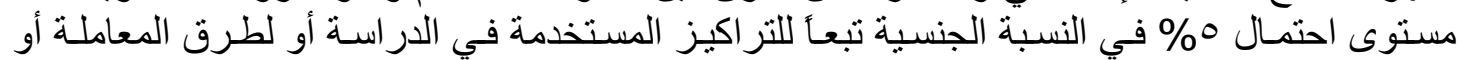
لدرجة الحرارة وللتداخل بينهما.

الجدول (r) : نأثثير تر اكيز مختلفة من التريكارد وطريقة المعاملة ودرجة الحرارة و التداخل فيما بينها في منوسط النسبة الجنسية للحشرة لجيلين متعاقبين

\begin{tabular}{|c|c|c|c|c|c|c|c|c|c|c|c|c|}
\hline \multicolumn{10}{|c|}{ متوسط النسبة الجنسية } & \multirow{4}{*}{$\begin{array}{c}3 \\
\frac{1}{3} \\
: 0 \\
0 \\
0\end{array}$} & \multirow{4}{*}{ 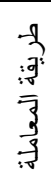 } & \multirow{4}{*}{$\begin{array}{l}\overline{7} \\
\overline{7} \\
\text { 。․ }\end{array}$} \\
\hline \multirow{2}{*}{\multicolumn{2}{|c|}{ لطريقة المعاملة }} & \multirow{2}{*}{\multicolumn{2}{|c|}{ للدرجة الحرارة }} & \multirow{2}{*}{\multicolumn{2}{|c|}{ 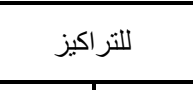 }} & \multicolumn{4}{|c|}{ للتناخل بين التزر اكيز وطريقة المعاملة ودرجة الحرارة } & & & \\
\hline & & & & & & \multicolumn{2}{|c|}{ المتوسط } & \multicolumn{2}{|c|}{ 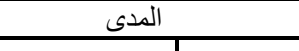 } & & & \\
\hline أنثى & ذكر & أنثى & 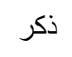 & أنثى & 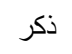 & أنثى & ذكر & أنثى & 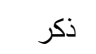 & & & \\
\hline \multirow[t]{4}{*}{$i_{i}^{\cdot r}$} & $\begin{array}{c}1.14 \\
1 \\
\end{array}$ & $i^{\cdot r}$ & $\begin{array}{c}1.14 \\
1\end{array}$ & 1.1 & ب & $\cdot \frac{+1}{i}$ & $\because{ }^{\prime}+1.1 \varepsilon$ & $1-1$ & $\begin{array}{r}-1.97 \\
1.4 .\end{array}$ & \multirow{8}{*}{ ro } & \multirow{4}{*}{ 需 } & 1 \\
\hline & & & & 1.1 & أب 17 & $\frac{ \pm 1}{i}$ & $\because .0+1.1 \varepsilon$ & $1-1$ & $\begin{array}{l}-1.97 \\
1.44\end{array}$ & & & $r$ \\
\hline & & & & $\begin{array}{l}1.4 \\
i\end{array}$ & أ & $\because \cdot \varepsilon+1 . \cdot \varepsilon$ & 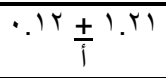 & $1.11-1$ & $1 . \leqslant 1-1$ & & & 0 \\
\hline & & & & $a_{i}^{1 . r}$ & 1.4 & $\because \cdot v^{\prime}+1 \cdot \cdot v$ & $\because \cdot \frac{1}{1} \cdot \cdot \cdot \varepsilon$ & $1 . Y r_{-1}$ & $1.11-1$ & & & $3=3$ \\
\hline \multirow[t]{8}{*}{$a^{1}$} & $i_{1}^{1.14}$ & & & & & $\because \cdot \frac{1}{\mathrm{i}}+1.1$ & $\because K \frac{1}{i}+r^{r}$ & $1 . \cdot \leq-1$ & $1 . \Gamma \wedge-1$ & & \multirow{4}{*}{$\overline{3}$} & 1 \\
\hline & & & & & & $\because \cdot 0+1.00$ & $\because \cdot r^{r} \pm 1 . r$ & $1.10-1$ & $1.7-1$ & & & r \\
\hline & & & & & & $\because \cdot v+\frac{1}{i} \cdot v$ & $\because{ }^{1}+1.11$ & $1 . Y_{-1}$ & $1 . Y V_{-1}$ & & & $\circ$ \\
\hline & & & & & & $\cdot \frac{ \pm}{i}$ & $\because \cdot \frac{ \pm}{1} \cdot \cdot \varepsilon$ & $1-1$ & $1.11-1$ & & & $?=$ \\
\hline & & $\begin{array}{l}1 \\
1\end{array}$ & 1.10 & & & $\cdot \frac{ \pm}{i}$ & $\because \mathrm{VV}+1.1 \mathrm{~V}$ & $1-1$ & $1.0 \cdot-1$ & \multirow{4}{*}{ r. } & \multirow{4}{*}{ 可 } & 1 \\
\hline & & & & & & $\because \cdots+1 \pm 1$ & $\because 1 \leq \frac{ \pm}{i} \mid r V$ & $1 .+r-1$ & $1 . \leqslant V_{-} 1$ & & & $r$ \\
\hline & & & & & & $\because \cdots+1+\cdot 1$ & $\because 0 \frac{0}{1} 1.1$. & $1 . \cdot \varepsilon-1$ & $1.10-1$ & & & $\circ$ \\
\hline & & & & & & $\cdot \frac{ \pm}{i}$ & $\cdot \frac{ \pm}{i}$ & $1-1$ & $1-1$ & & & $\because=$ \\
\hline
\end{tabular}




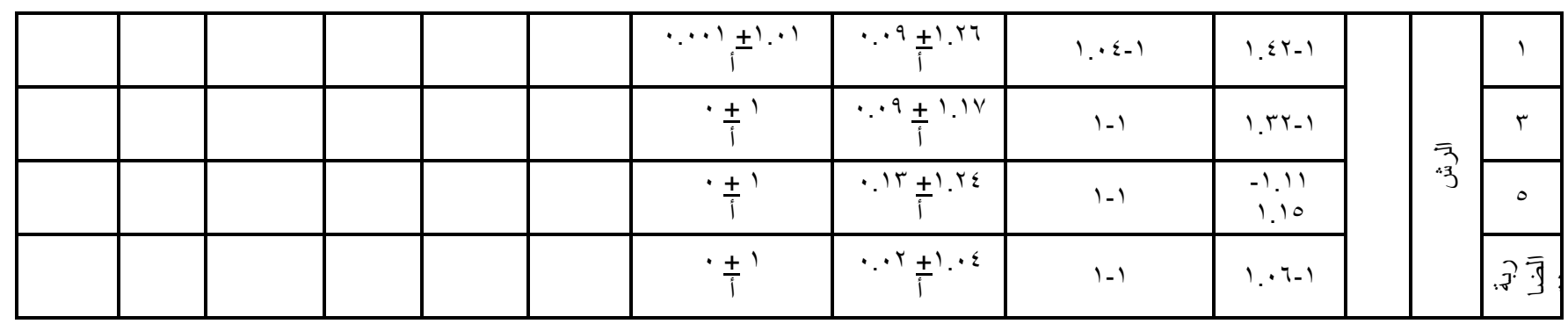

* المتوسطات ذات الأحرف غير المنشابهة تشير إلى وجود فروقات معنوية عند مستوى احتمال 0\%.

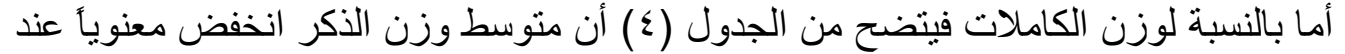

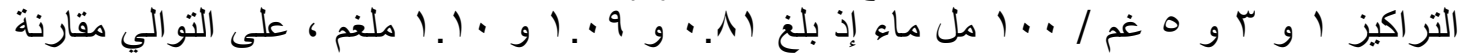

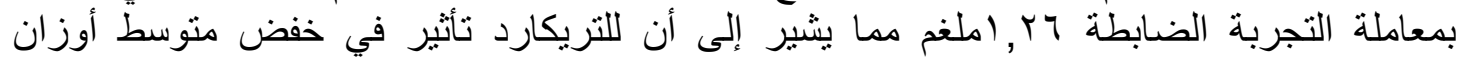

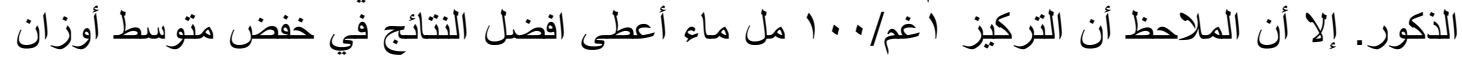

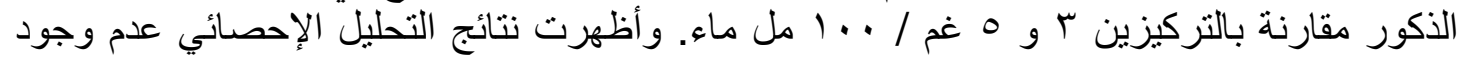

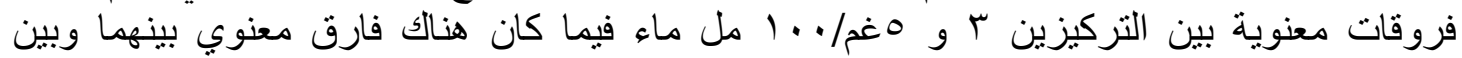

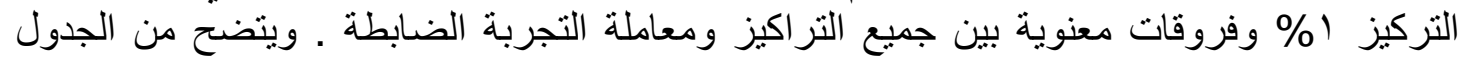

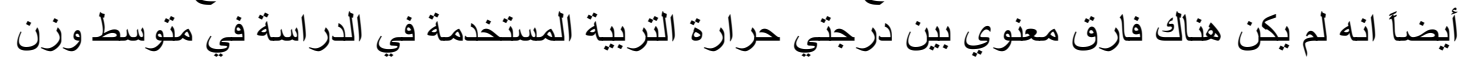

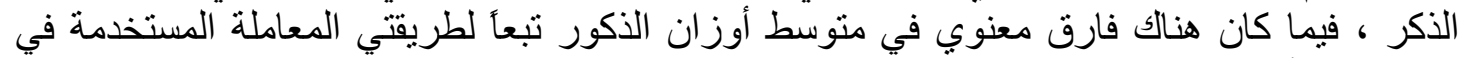

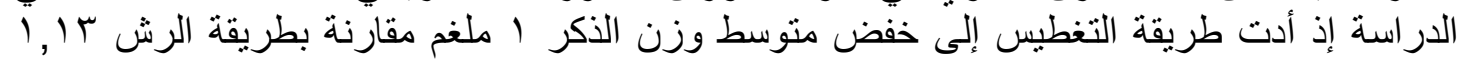

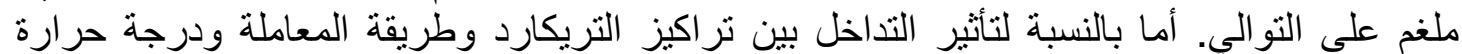

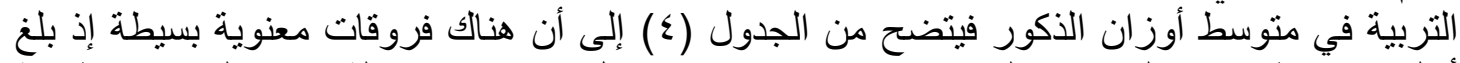

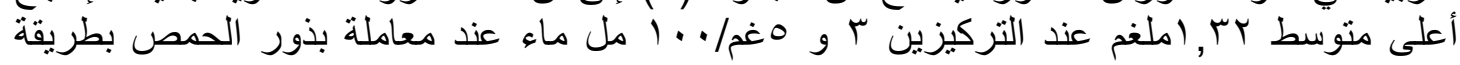

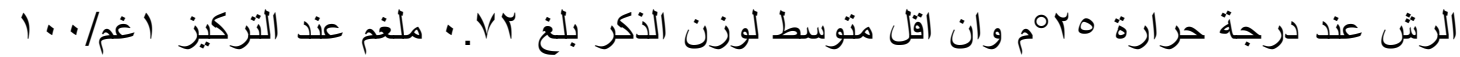

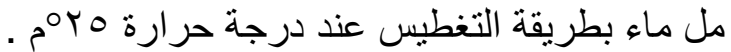

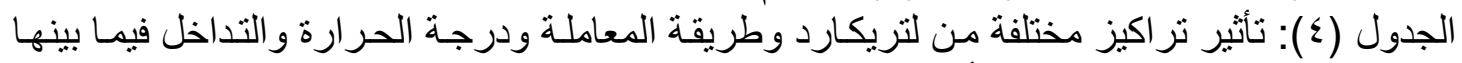
في المتوسط العام لأوزان الذكور لجيلين متعاقبين

\begin{tabular}{|c|c|c|c|c|c|c|c|}
\hline \multicolumn{5}{|c|}{ متوسط وزن الذكور/ملغم } & \multirow{3}{*}{ الحر درجة } & \multirow{3}{*}{ المعايقة } & \multirow{3}{*}{ التر اكيز\% } \\
\hline \multirow{2}{*}{ الطريقة } & \multirow{2}{*}{ 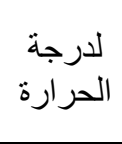 } & \multirow{2}{*}{ للتزر اكيز } & \multicolumn{2}{|c|}{ للتداخل بين التر اكيز وطريقة المعاملة } & & & \\
\hline & & & S.E المتوسط & 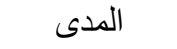 & & & \\
\hline \multirow[t]{4}{*}{ i) } & 11.9 & $\{\cdot .11$ & $i \cdot . \cdot \wedge_{ \pm} \cdot v r$ & $.10-.00$ & \multirow{8}{*}{ ro } & \multirow{4}{*}{ التغطيس } & 1 \\
\hline & & 9 9 & & $1.1 \cdot \cdots . \wedge$ & & & $r$ \\
\hline & & 1.1.اب & & $1.1 \cdots . \wedge$ & & & 0 \\
\hline & & $\rightarrow 1.17$ & $j g \cdot . \cdot r \pm 1 . r r$ & $1 . \varepsilon \cdot-1 . \mu \cdot$ & & & التجربة الضابطة \\
\hline \multirow[t]{12}{*}{ (1) } & & & 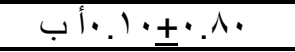 & $\cdot .90-.7$. & & \multirow{4}{*}{ الرش } & 1 \\
\hline & & & jو & $1 . r 0-1.40$ & & & $r$ \\
\hline & & & rr. & $1.0 .-1.10$ & & & $\circ$ \\
\hline & & & jه & $1 . r \cdot-1 . r \cdot$ & & & التجربة الضـابطة \\
\hline & $11 . .0$ & & & $1-\cdot .10$ & \multirow{8}{*}{ r. } & \multirow{4}{*}{ التغطيس } & 1 \\
\hline & & & 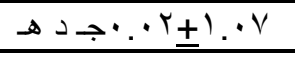 & $1.1 \cdot-1.0$ & & & $\Gamma$ \\
\hline & & & & $1.10-.90$ & & & 0 \\
\hline & & & & $1.0-1$ & & & التجربة الضابطة \\
\hline & & & . & $\because .90-.0$ & & \multirow{4}{*}{ الرش الرش } & 1 \\
\hline & & & ج & $1 . r \cdot \cdots . \Lambda \cdot$ & & & $\Gamma$ \\
\hline & & & 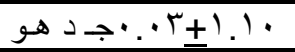 & $1.10-1.0$ & & & 0 \\
\hline & & & $j \cdot .9 \pm \pm 1 . \varepsilon r$ & $1.00-1.50$ & & & التجربة الضـابطة \\
\hline
\end{tabular}

* المتوسطات ذات الأحرف غير المنشابهة تشير إلى وجود فروقات معنوية عند مستوى احتمال ه\%. 


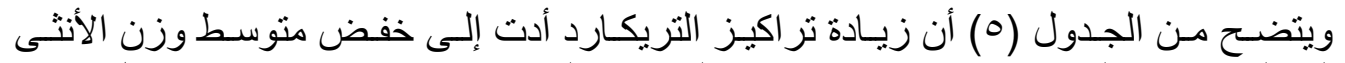

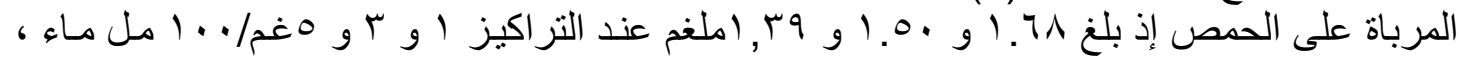

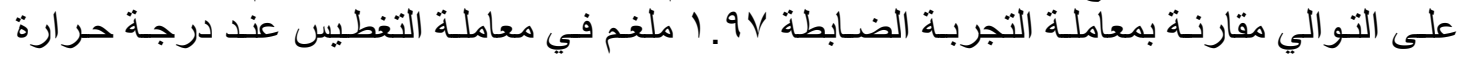

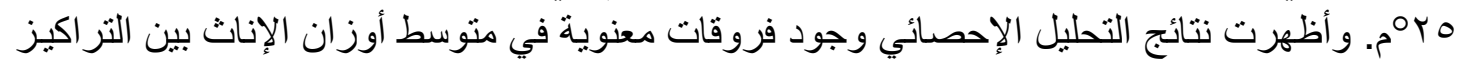

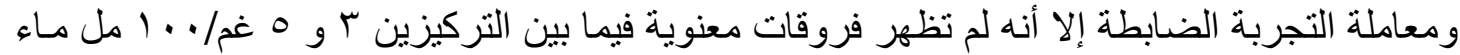

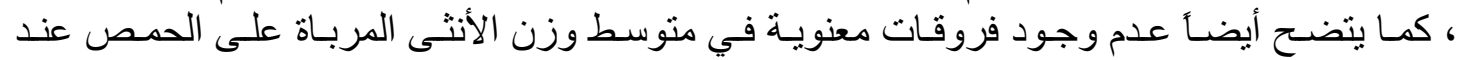

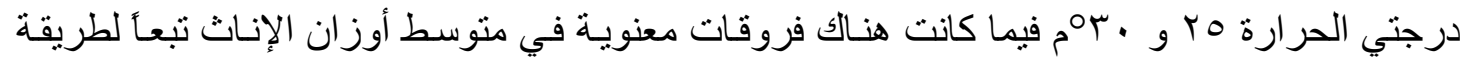

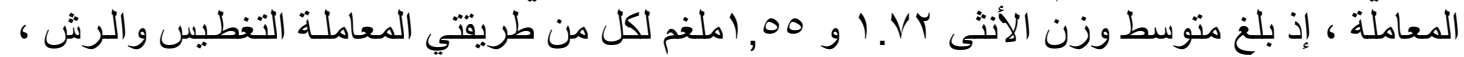

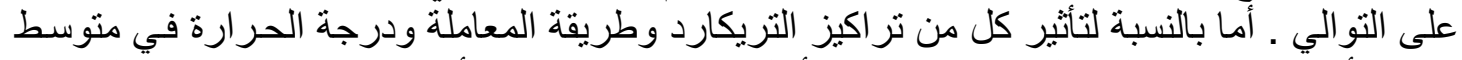

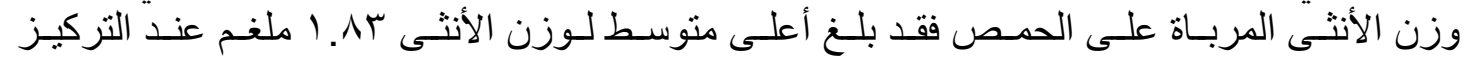

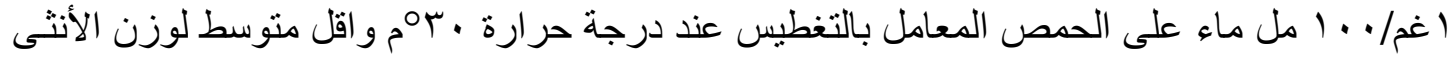

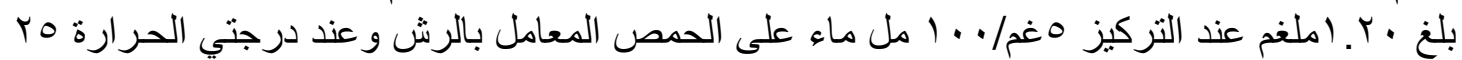

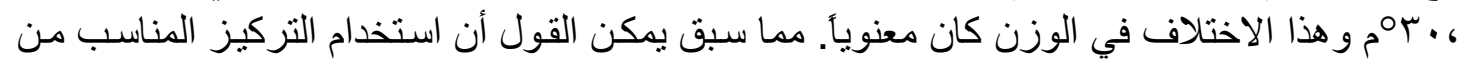

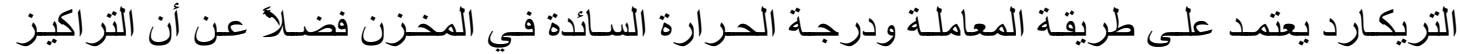
الواطئة من المبيد يمكن أن يكون لها تأثير غير مباشر في خفض الثرة أعداد الحشرة في المخزن.

الجدول (0): تأثير تر اكيز مختلفة من تريكارد وطريقة المعاملة ودرجة الحر ارة و التداخل فيما بينها في المتوسط العام لأوزان الإناث لجيلين متعاقيبين

\begin{tabular}{|c|c|c|c|c|c|c|c|}
\hline \multicolumn{5}{|c|}{ متوسط وزن الإناث/ملغم } & \multirow{3}{*}{ الحرارة } & \multirow{3}{*}{ طريقة } & \multirow{3}{*}{ التر اكيز\%\% } \\
\hline \multirow[t]{2}{*}{ الطريقة } & \multirow{2}{*}{ 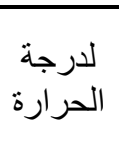 } & \multirow[t]{2}{*}{ ل لتر اكيز } & \multicolumn{2}{|c|}{ 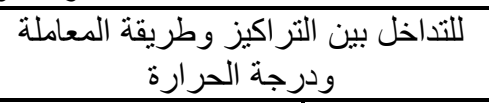 } & & & \\
\hline & & & S.E المتوسط & المدى & & & \\
\hline \multirow[t]{4}{*}{ بr } & 11.74 & (ب & & $1 . V-1 . \varepsilon$ & ro & التغطيس & 1 \\
\hline & & $i 1.0$ & 的. & $1.0-1.2$ & \multirow{7}{*}{ 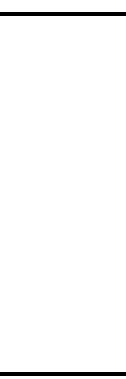 } & \multirow[t]{3}{*}{ 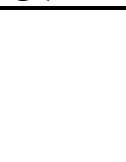 } & $r$ \\
\hline & & 11.19 & 的. & $1.0-1.2$ & & & 0 \\
\hline & & $\rightarrow 1.9 v$ & $\Delta \cdot .1 T_{ \pm} r^{\prime} \cdot r$ & r.ro-1.10 & & & التجربة الضابطة \\
\hline \multirow[t]{12}{*}{11.00} & & & $د{ }^{\prime} \cdot .9 \pm 1 . V r$ & $1.9-1.7$ & & \multirow{4}{*}{ الرش } & 1 \\
\hline & & & & $1.70-1 . \varepsilon$ & & & $r$ \\
\hline & & & $i \cdot .1 \pm 1 . r$ & $1 . \Gamma-1$ & & & 0 \\
\hline & & & 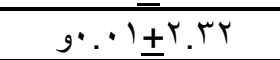 & Y.rO-Y.r & & & التجربة الضابطة \\
\hline & $11.7 r$ & & 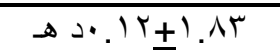 & $r-1.7$ & $r$. & التغطيس & 1 \\
\hline & & & $2+.11 \pm 1.70$ & $1.10-1.0$ & & & $r$ \\
\hline & & & د & $1 . V-1 . V$ & & & 0 \\
\hline & & & $g-. \cdot V_{ \pm} r^{\prime} \cdot \Lambda$ & $5.10-1.90$ & & & التجربة الضابطة \\
\hline & & & د & $1.90-1.50$ & & & 1 \\
\hline & & & & $1.0-1 . r$ & & & $r$ \\
\hline & & & $1 \cdot . \cdot 7 \pm 1 . r$ & $1, r-1,1$ & & الرس & 0 \\
\hline & & & & $1.7-1.40$ & & & التجربة الضابطة \\
\hline
\end{tabular}

* المتوسطات ذات الأحرف غير المتشابهة تشير إلى وجود فروقات معنوية عند مستوى احتمال ٪\% 


\title{
BIOLOGICAL ACTIVITY OF Callosobruchus maculatus (Fab.) REARED ON CHICKPEA
}

Nazar M. Al-Mallah Fahad A. A. Al-Mekhlafi

Plant Prot. Dept., College of Agric. and Forestry, Mosul Univ., Mosul-Iraq

\begin{abstract}
The results of the study revealed that the interaction between Trigard 75\% W.P. concentrations, treatment methods and insect rearing temperatures on Callosobruchus maculatus reared on chickpea Cicer arietinum (L.) exhibit a varied effect on the rate of insect increase and dipping the chickpea seeds for one minute in a solution of Trigard $5 \%$ at $30 \mathrm{C}^{\circ}$, reduce the average of increase rate to 1.37 in comparison with control which reached 34.73 . The effect of interaction between Tigard concentration, treatment methods and insect rearing temperature on the average of food consumption and the average generation period for two generations showed that increasing Trigard concentrations coincide with the reducing in the average weight loss of food of chickpea. Dipping the chickpea seeds in Trigard solution protect the seeds from insect attack in comparison with seed sprayed by Trigard and increasing the Tigard concentration increased the average generation period 34.17 day which was obtained from seed sprayed by $5 \%$ Trigard at $25 \mathrm{C}^{\circ}$. The studied factors showed no effect on sex ratio while it showed a significant difference for the weight between males and females.
\end{abstract}

A part of Msc. Thesis of the second author 


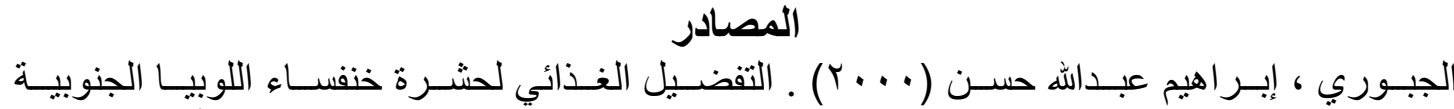

Callosobruchus maculatus (F) Bruchidae: Coleoptera

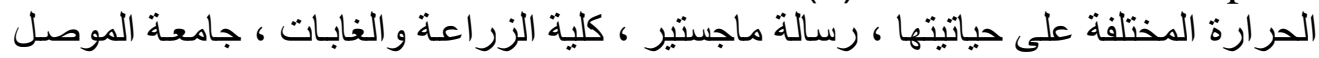

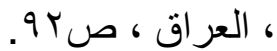

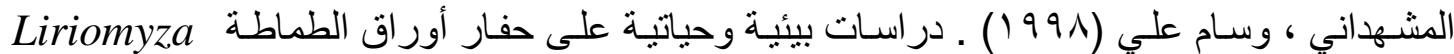

bryoninia (kalt)

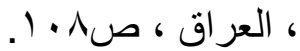

Berry, E. C; A. A. Favagalla; and W. D. Guthrle (1980). Field evaluation of Diflubenzuron for control of first and second. generation of european corn borerr. J. Econ. Entomol. 73: 634-636.

Buholzer, F; J. Draber; F. Bourgeois ; and W. Guyer (1992). CGA, 184; 699 a new acylurea insecticide. Med. Fac. Landbouw. Univ. Cent. 57 (3): 790.

Busvine, J.R. (1971). Acritical review of the techniques for testing insecticides. Commonwealth Agricultural Bureax Dorset Press, London. pp. 345.

Hallak, H. (1993) Effect of elevated temperature on the growth and reproduction of cowpea weevil $C$. maculatus $(\mathrm{F})$ Coleoptera: Bruchidae and it use a factor to reduce their damage to stored grains. Arab J. of Plant Protection11(2): 66-72.

Ishimoto M; T. Sato; M. Chrispeels; and S. kitamurak (1996). Bruchid resistance of transgenic azuki bean expressing seed amylase inhibitor of common bean. J. Entomolgia Experimentalis et Applicata 79: 309-315.

Krebs J; (1978). The experimental analysis of distribution and abundance. Harper and Row Publishers. New York, USA.

Prevett, P. F. (1961). Field Infestation of Cowpea Pads by Beetles of Burchidae and Curculionidae in Northern Nigeria. Bull. Ent. Res. 52: 635-645.

Saito, T. (1992). Effect of insecticides on Liriomyza trifolii (Burg). Proc. Plant Protection $46: 181-191$.

Williams M. C; (1967). Third generation of pesticide. Scientific America, W.H. Freeman and Company, San Francisco, USA. 